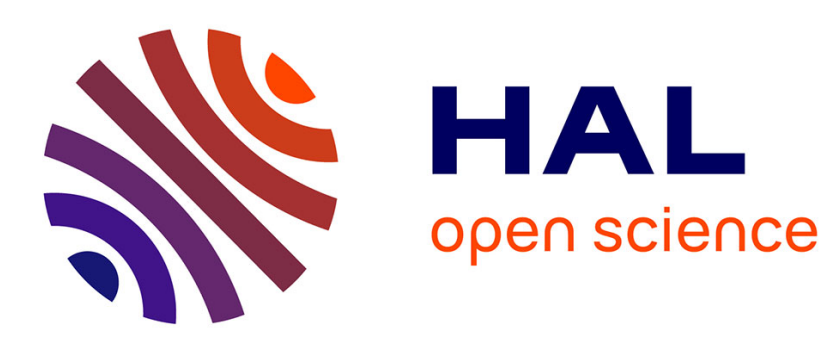

\title{
Counterexample to a Lyapunov Condition for Uniform Asymptotic Partial Stability
}

Jakub Orlowski, Antoine Chaillet, Mario Sigalotti

\section{To cite this version:}

Jakub Orlowski, Antoine Chaillet, Mario Sigalotti. Counterexample to a Lyapunov Condition for Uniform Asymptotic Partial Stability. IEEE Control Systems Letters, 2020, 4 (2), pp.397-401. 10.1109/LCSYS.2019.2939717 . hal-02291650

\section{HAL Id: hal-02291650 \\ https://hal.science/hal-02291650}

Submitted on 16 Mar 2020

HAL is a multi-disciplinary open access archive for the deposit and dissemination of scientific research documents, whether they are published or not. The documents may come from teaching and research institutions in France or abroad, or from public or private research centers.
L'archive ouverte pluridisciplinaire HAL, est destinée au dépôt et à la diffusion de documents scientifiques de niveau recherche, publiés ou non, émanant des établissements d'enseignement et de recherche français ou étrangers, des laboratoires publics ou privés. 


\title{
Counterexample to a Lyapunov condition for uniform asymptotic partial stability
}

\author{
Jakub Orłowski ${ }^{1}$, Antoine Chaillet ${ }^{2}$, and Mario Sigalotti ${ }^{3}$
}

\begin{abstract}
Partial stability characterizes dynamical systems for which only a part of the state variables exhibits a stable behavior. In his book on partial stability, V. I. Vorotnikov proposed a sufficient condition to establish this property through a Lyapunov-like function whose total derivative is upper-bounded by a negative definite function involving only the sub-state of interest. In this note, we show with a simple two-dimensional system that this statement is wrong in general. More precisely, we show that the convergence rate of the relevant state variables may not be uniform in the initial state. We also discuss the impact of this lack of uniformity on the connected issue of robustness with respect to exogenous disturbances.
\end{abstract}

\section{INTRODUCTION}

In many control applications, it is relevant to impose an appropriate behavior only on a part of the state variables. Several properties are suited for this kind of analysis, such as partial stability [14], stability with respect to two measures [6], or input-to-output stability (IOS, [11]). These properties can be established using powerful Lyapunov characterizations [13], [12], even in a time-delay context [3], [2].

Questions of partial stability arise naturally in adaptive control, where the state of the system is extended to include adaptive variables, which do not necessarily have to fulfill strict stability conditions.

Partial stability has been thoroughly described in the seminal book by V. I. Vorotnikov [14], and later revisited in his overview article [15]. One of the theorems therein [14, Theorem 6.2.1] introduces a seemingly powerful Lyapunov tool to establish partial stability of nonlinear time-delay systems. This statement claims that partial stability can be guaranteed using a Lyapunov-like functional with a dissipation rate depending on the output norm only. This result also states that partial stability

\footnotetext{
*This work was supported by Digicosme and IRS iCODE.

${ }^{1}$ Jakub Orłowski is with L2S-CentraleSupélec-Univ. Paris Saclay. jakub.orlowskiel2s.centralesupelec.fr

${ }^{2}$ Antoine Chaillet is with L2S-CentraleSupélecUniv. Paris Saclay and Institut Universitaire de France. antoine.chailletecentralesupelec.fr

${ }^{3}$ Mario Sigalotti is with Inria \& Laboratoire Jacques-Louis Lions, Sorbonne Université, Univ. Paris-Diderot SPC, CNRS. mario.sigalotti@inria.fr
}

is uniform in the initial state, meaning that a common convergence rate is ensured for all initial states in a given neighborhood of the origin.

In [8], we have used this theorem to prove that an adaptive control law with $\sigma$-modification [1] leads to oscillation quenching in a nonlinear time-delay model representing two interconnected populations of neurons, and that steady-state oscillations amplitude is proportional (up to a comparison function) to the value of the tuning parameter $\sigma$.

Upon closer inspection, however, we have noticed that the claims made by [14, Theorem 6.2.1] are stronger than what actually follows from the premises. Namely, the result does guarantee partial asymptotic stability but the convergence rate is not necessarily uniform in the initial state. To the best of our knowledge, this error has never been mentioned in the literature.

This paper provides a simple counterexample to [14, Theorem 6.2.1-(5)] through a two-dimensional delayfree system. It also underlines the consequences of the lack of uniformity with respect to initial state in the context of robustness analysis. Namely, we show though a simple example that (non-uniform) partial asymptotic stability does not necessarily ensure converging input converging output property, even for systems evolving on a bounded set.

The remainder of the paper is organized as follows. In Section II we recall the definition of partial stability from [14], and the theorem in question, which we also reformulate in a non-delayed time-invariant context. In Section III we present the counterexample to the theorem. In Section IV we investigate the relation between uniform partial asymptotic stability and input-to-output stability for systems evolving on a bounded set, and discuss the importance of uniformity in this context.

Notation. Given $x \in \mathbb{R}^{n},|x|$ denotes its Euclidean norm. Given an interval $I \subset \mathbb{R}$ and a locally essentially

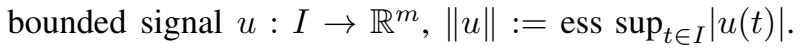
Given $X \subset \mathbb{R}^{n}, C(I, X)$ denotes the set of all continuous functions $\phi: I \rightarrow X$ and, given $\bar{\delta}>0$, $\mathcal{C}:=C([-\bar{\delta}, 0], \mathbb{R})$. Given a continuous signal $x$ : 
$[-\bar{\delta},+\infty) \rightarrow \mathbb{R}^{n}$ and a time $t \geq 0, x_{t} \in \mathcal{C}^{n}$ denotes the history function defined as $x_{t}(s):=x(t+s)$ for all $s \in[-\bar{\delta}, 0]$. A function $\alpha: \mathbb{R}_{\geq 0} \rightarrow \mathbb{R}_{\geq 0}$ is said to be of class $\mathcal{K}$ if it is continuous, increasing, and zero at zero. A function $\beta: \mathbb{R}_{\geq 0} \times \mathbb{R}_{\geq 0} \rightarrow \mathbb{R}_{\geq 0}$ is of class $\mathcal{K} \mathcal{L}$ if $\beta(\cdot, t) \in \mathcal{K}$ for each $t \in \mathbb{R}_{\geq 0}$ and, for each $s \in \mathbb{R}_{\geq 0}$, $\beta(s, \cdot)$ is continuous, non-increasing and tends to zero as its argument tends to infinity.

\section{PARTial StaBility}

\section{A. Definitions}

We start by recalling basic notions of partial stability of nonlinear time-delay systems. We deliberately follow the notation and terminology used in [14] as closely as possible. Consider a time-delay system of the form

$$
\dot{x}(t)=X\left(t, x_{t}\right),
$$

with state $x_{t} \in \mathcal{C}^{n} . x_{t}$ is decomposed as $x_{t}=\left(y_{t}, z_{t}\right)$, where $y_{t} \in \mathcal{C}^{m}, m \leq n$, represents the state variables of interest and $z_{t} \in \mathcal{C}^{n-m}$. The vector field $X$ : $\mathbb{R}_{\geq 0} \times \mathcal{C}^{n} \rightarrow \mathbb{R}^{n}$ is assumed continuous and satisfying the conditions for existence and uniqueness of solutions in the domain

$$
\left\{\left(t, x_{t}\right) \mid t \geq 0,\left\|y_{t}\right\|<H,\left\|z_{t}\right\|<\infty\right\},
$$

with $z$-continuable solutions, that is, all solutions are defined for all $t \geq t_{0}$ such that $\left|y\left(t ; t_{0}, x_{0}\right)\right|<H$. Here $x_{0}=\left(y_{0}, z_{0}\right) \in \mathcal{C}^{n}$ is the initial state of the system, $x\left(t ; t_{0}, x_{0}\right)$ is the solution of (1) at time $t$, starting from $x_{0}$ at time $t_{0}$, and $y\left(t ; t_{0}, x_{0}\right)$ is the $y$ part of $x\left(t ; t_{0}, x_{0}\right)$. We will make use of the following representation of (1):

$$
\begin{aligned}
& \dot{y}(t)=Y\left(t, y_{t}, z_{t}\right) \\
& \dot{z}(t)=Z\left(t, y_{t}, z_{t}\right) .
\end{aligned}
$$

The following two definitions of partial stability are taken from [14, Definition 6.1.1].

Definition $1(y-A S)$ : The zero solution $x(\cdot) \equiv 0$ of (1) is said to be asymptotically $y$-stable $(y-\mathrm{AS})$ if

- it is $y$-stable, that is, for any numbers $\varepsilon>0, t_{0} \geq 0$, there exists a number $\delta\left(\varepsilon, t_{0}\right)>0$ such that

$$
\left\|x_{0}\right\|<\delta \Longrightarrow\left|y\left(t ; t_{0}, x_{0}\right)\right|<\varepsilon, \quad \forall t \geq t_{0} ;
$$

- for each $t_{0} \geq 0$, there exists a number $\Delta\left(t_{0}\right)>$ 0 such that solutions $x\left(\cdot ; t_{0}, x_{0}\right)$ with $\left\|x_{0}\right\|<\Delta$ satisfy

$$
\lim _{t \rightarrow \infty}\left|y\left(t ; t_{0}, x_{0}\right)\right|=0 .
$$

Definition 2 (y-UAS): The zero solution $x(\cdot) \equiv 0$ of (1) is said to be uniformly asymptotically $y$-stable ( $y$ UAS) if
- it is $y$-stable uniformly in $t_{0}$, that is, for any $\varepsilon>0$, there exists a $\delta(\varepsilon)>0$ such that from $\left\|x_{0}\right\|<\delta$ and any $t_{0} \geq 0$ it follows that $\left|y\left(t ; t_{0}, x_{0}\right)\right|<\varepsilon$ for all $t \geq t_{0}$,

- there exists a number $\Delta>0$ such that, given any $t_{0} \geq 0$, solutions $x\left(\cdot ; t_{0}, x_{0}\right)$ with $\left\|x_{0}\right\|<\Delta$ satisfy the condition

$$
\lim _{t \rightarrow \infty}\left|y\left(t ; t_{0}, x_{0}\right)\right|=0,
$$

- relationship (5) holds uniformly with respect to $t_{0}$ and $x_{0}$ from the domain $t_{0} \geq 0,\left\|x_{0}\right\|<\Delta$, that is, for every $\varepsilon>0$ there exists $T(\varepsilon)>0$ such that, for all $t_{0} \geq 0$,

$$
\left\|x_{0}\right\| \leq \Delta \Longrightarrow\left|y\left(t ; t_{0}, x_{0}\right)\right| \leq \varepsilon, \quad \forall t>t_{0}+T .
$$

Following the terminology of [9], the zero solution would be called asymptotically $y$-stable uniformly in $\left\{t_{0}, x_{0}\right\}$, which better highlights that the qualitative behavior of solutions (in terms of convergence rate and transient overshoot) is uniform in both the initial time $t_{0}$ and the initial state $x_{0}$.

\section{B. Disproved sufficient condition}

Given any locally Lipschitz function $V: \mathbb{R}_{\geq 0} \times \mathcal{C}^{n} \rightarrow$ $\mathbb{R}_{\geq 0}$, let $\dot{V}$ denote its upper-right Dini derivative along the solutions of (1):

$$
\dot{V}\left(t, x_{t}\right):=\varlimsup_{h \rightarrow 0^{+}} \frac{V\left(t+h, x_{t+h}\right)-V\left(t, x_{t}\right)}{h} .
$$

Furthermore, given $\phi \in \mathcal{C}^{n}$, let $\phi_{y} \in \mathcal{C}^{m}$ and $\phi_{z} \in$ $\mathcal{C}^{n-m}$ be such that $\phi=\left(\phi_{y}, \phi_{z}\right)$.

In his book [14], V. I. Vorotnikov states the following.

Assertion 3 (Theorem 6.2.1-(5) in [14], disproved): Suppose that for (1) it is possible to specify a function $V=V(t, \phi)$, locally Lipschitz in domain (2), such that

$$
\begin{aligned}
a\left(\left|\phi_{y}(0)\right|\right) & \leq V(t, \phi) \leq b(\|\phi\|), \\
\dot{V}\left(t, x_{t}\right) & \leq-c(|y(t)|), \\
|Y(t, \phi)| & \leq M=\mathrm{const}>0
\end{aligned}
$$

where $a, b$, and $c$ are class $\mathcal{K}$ functions. Then the zero solution of (1) is uniformly asymptotically $y$-stable.

This result is similar to the classical LyapunovKrasovskii characterization of asymptotic stability for time-delay systems [5, Theorem 31.1] when $x=y$. As shown in [9, Theorem 15] and [6, Theorem 3.1.3], the above assumptions do assure $y$-AS. Assertion 3 seemingly generalizes these results by guaranteeing uniformity in the initial state. What makes this result particularly appealing is that both the lower bound on 
$V$ and its dissipation rate are allowed to involve merely the state variables of interest $y$, as opposed to classical results on output stability that require dissipation in terms of the whole functional $V$ [12], [2], [3], [13].

If the conditions of Assertion 3 are met, the zero solution is indeed $y$-AS. The proof of Theorem 6.2.1(5) in [14] shows $y$-stability and asymptotic convergence of $y$ and then it proceeds to conclude that $y$-UAS follows from these premises. However, in Section III, we show that convergence may not be uniform in $x_{0}$.

In order to lighten the notation, let us write down an immediate corollary to Assertion 3, for the particular case of time-invariant non-delayed dynamics, namely:

$$
\begin{aligned}
& \dot{y}(t)=Y(y(t), z(t)), \\
& \dot{z}(t)=Z(y(t), z(t)),
\end{aligned}
$$

where $x(t)=(y(t), z(t)) \in \mathbb{R}^{n}, Y: \mathbb{R}^{n} \rightarrow \mathbb{R}^{m}, Z$ : $\mathbb{R}^{n} \rightarrow \mathbb{R}^{n-m}$ are continuous and satisfy the conditions for existence and uniqueness of solutions in the domain

$$
\{x=(y, z)|| y|<H,| z \mid<\infty\} .
$$

Assertion 4 (Disproved): Suppose there exists a locally Lipschitz function $V: \mathbb{R}^{n} \rightarrow \mathbb{R}_{\geq 0}$ such that, in the domain (12),

$$
\begin{aligned}
a(|y|) & \leq V(x) \leq b(|x|), \\
\dot{V}(x(t)) & \leq-c(|y(t)|), \\
|Y(x)| & \leq M,
\end{aligned}
$$

where $a, b$, and $c$ are class $\mathcal{K}$ functions and $M>0$. Then the zero solution of (11) is $y$-UAS.

We stress that, since the considered class of systems is time-invariant, uniformity in $t_{0}$ follows automatically.

\section{CountereXAmple}

In this section we disprove [14, Theorem 6.2.1-(5)] by constructing a system that fulfills the hypotheses of Assertion 3 but is not $y$-UAS.

As we detail below, the uniformity in our counterexample is compromised by a stickiness effect of the equilibrium, which means that solutions starting from an initial state with $y_{0}$ close to the equilibrium take arbitrarily long to go through their transient behavior. It should be noted that it is not possible to find such a system when $y=x$. Indeed, for time-invariant finitedimensional systems, asymptotic stability implies uniform asymptotic stability [7, Theorem 7 (e)].

Consider the system

$$
\begin{aligned}
& \dot{y}(t)=-\operatorname{sat}(z(t) y(t)), \\
& \dot{z}(t)=\operatorname{sat}\left(y(t)^{2}\right),
\end{aligned}
$$

where $x(t)=(y(t), z(t)) \in \mathbb{R}^{2}$, and sat denotes the classical saturation function:

$$
\operatorname{sat}(s):=\min \{|s|, 1\} \operatorname{sign}(s), \quad \forall s \in \mathbb{R} .
$$

This system has the form (11) and condition (15) holds with $M=1$ for all $x \in \mathbb{R}^{2}$. Moreover, the vector field $X=(Y, Z)^{T}$ is globally Lipschitz, so we get existence and uniqueness of solutions for all times $t \geq 0$ and all initial states $x_{0} \in \mathbb{R}^{2}$.

Proposition 5: System (16) with the Lyapunov function $V(x):=2|x|-z$ fulfills all the conditions of Assertion 4 (hence, Assertion 3) but is not $y$-UAS.

Proof: The Lyapunov function $V$ can be written explicitly as

$$
V(x)=2 \sqrt{y^{2}+z^{2}}-z .
$$

It is locally Lipschitz on $\mathbb{R}^{2}$ and 0 at 0 . Since $|z| \leq|x|$, it holds that $V(x) \leq 3|x|$ for all $x \in \mathbb{R}^{2}$. Moreover,

$$
\frac{\sqrt{2}}{2}(|y|+|z|) \leq \sqrt{y^{2}+z^{2}} \leq|y|+|z|,
$$

which implies that

$$
\begin{aligned}
V(x) & \geq \sqrt{2}(|y|+|z|)-z \\
& \geq(\sqrt{2}-1)(|y|+|z|) \\
& \geq(\sqrt{2}-1) \sqrt{y^{2}+z^{2}} .
\end{aligned}
$$

It follows that

$$
a(|y|) \leq a(|x|) \leq V(x) \leq b(|x|), \quad \forall x \in \mathbb{R}^{2},
$$

where $a(s):=(\sqrt{2}-1) s$ and $b(s):=3 s$, for all $s \geq 0$. We conclude that (13) holds for all $x \in \mathbb{R}^{2}$.

Now, assume that $\left|x_{0}\right|<1$. Then, as long as $|x(t)| \leq 1$, the solution of (16) coincides with that of

$$
\begin{aligned}
& \dot{y}(t)=-z(t) y(t), \\
& \dot{z}(t)=y(t)^{2} .
\end{aligned}
$$

Consider the function $W(x):=|x|^{2}$. It can be easily seen that $\dot{W}(x(t))=0$ at all times, along the solutions of (18), so $t \mapsto W(t)$ is a first integral for (18). It follows in particular that $\left|x_{0}\right|<1$ implies $|x(t)|<1$ for all $t \geq 0$. This, in turn, ensures that, for all $\left|x_{0}\right|<1$, solutions of (16) coincide at all times with those of (18), and we have

$$
y(t)^{2}+z(t)^{2}=y_{0}^{2}+z_{0}^{2}, \quad \forall t \geq 0 .
$$

The derivative of $V$ along solutions of (16) reads

$$
\begin{aligned}
\dot{V}(x(t)) & =\frac{2\left(-z(t) y(t)^{2}+z(t) y(t)^{2}\right)}{\sqrt{y(t)^{2}+z(t)^{2}}}-y(t)^{2} \\
& =-y(t)^{2} .
\end{aligned}
$$




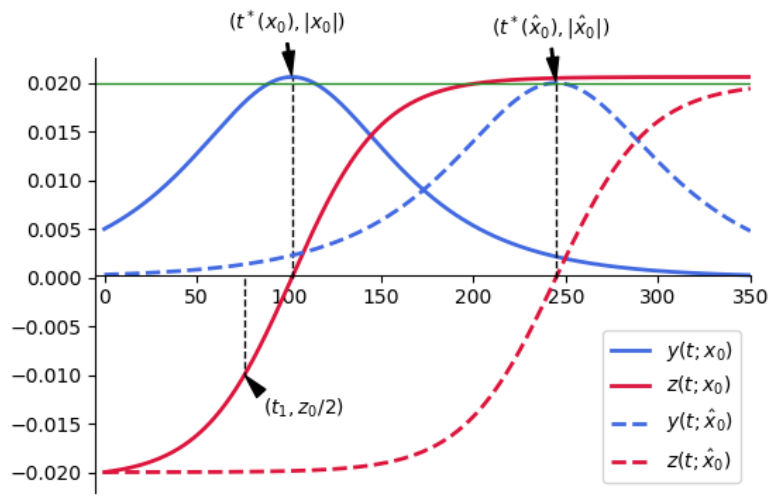

Fig. 1. Numerically simulated solutions of (16) with initial values $x_{0}=\left(5.10^{-3},-2.10^{-2}\right)$ (solid lines) and $\hat{x}_{0}=$ $\left(3.10^{-4},-2.10^{-2}\right)$ (dashed lines). At time $t^{*}\left(x_{0}\right)$, we have $z\left(t^{*} ; x_{0}\right)=0$ and $y\left(t^{*} ; x_{0}\right)=\left|x_{0}\right|$, its maximum value. Note that this value is always above $\left|z_{0}\right|$ (green line). Time $t_{1}\left(x_{0}\right)$, used in the proof, is such that $z\left(t_{1} ; x_{0}\right)=z_{0} / 2$. These simulations illustrate that a smaller value of $y_{0}$ makes the $y$ subsystem reach its maximum value at a later time.

In other words, $\dot{V}(x(t)) \leq-c(|y(t)|)$ on the domain (12) with $H=1$ and $c(s):=s^{2}$ for all $s \geq 0$. Thus, all the conditions of Assertion 4 (and hence, Assertion 3) are fulfilled.

To prove that uniform convergence to 0 does not hold, consider an $\left|x_{0}\right| \leq 1$ with $z_{0}<0$ and $y_{0}>0$. Then $y(t) \geq 0$ at all times. Moreover, since $z(t)$ is non-decreasing, $y(t)$ increases over $\left[0, t^{*}\right)$ and decreases over $\left(t^{*},+\infty\right)$, where $t^{*}=t^{*}\left(x_{0}\right)$ is the time at which $z\left(t^{*}\right)=0$. Furthermore, in view of (19), it holds that

$$
y\left(t^{*}\right)=\sqrt{y_{0}^{2}+z_{0}^{2}}=\left|x_{0}\right| .
$$

See Figure 1 for illustration. Let $t_{1}$ be the time at which $z\left(t_{1}\right)=\frac{z_{0}}{2}$. Then $t^{*}>t_{1}$, since $z_{0}<0$ and $z(t)$ is nondecreasing. Estimating $t_{1}$ will therefore provide a lower bound on $t^{*}$. To that aim, observe that since $z(t) \in$ $\left[z_{0}, \frac{z_{0}}{2}\right]$ for all $t \in\left[0, t_{1}\right]$, we get from (18a) that $\dot{y}(t) \leq$ $\left|z_{0}\right| y(t)$, which implies that

$$
y(t) \leq y_{0} e^{\left|z_{0}\right| t}, \quad \forall t \in\left[0, t_{1}\right] .
$$

Hence, for all $t \in\left[0, t_{1}\right]$,

$$
\dot{z}(t)=y(t)^{2} \leq y_{0}^{2} e^{2\left|z_{0}\right| t} .
$$

It follows that

$$
z(t) \leq z_{0}+y_{0}^{2} \int_{0}^{t} e^{2\left|z_{0}\right| \tau} \mathrm{d} \tau=z_{0}+y_{0}^{2}\left(\frac{e^{2\left|z_{0}\right| t}-1}{2\left|z_{0}\right|}\right)
$$

for all $t \in\left[0, t_{1}\right]$. Since $t_{1}$ is such that $z\left(t_{1}\right)=z_{0} / 2$, it is thus necessarily greater than the time $t_{2}$ for which

$$
\frac{z_{0}}{2}=z_{0}+y_{0}^{2}\left(\frac{e^{2\left|z_{0}\right| t_{2}}-1}{2\left|z_{0}\right|}\right) \text {, }
$$

meaning that

$$
t_{1} \geq t_{2}=\frac{1}{2\left|z_{0}\right|} \ln \left(1+\frac{z_{0}^{2}}{y_{0}^{2}}\right) .
$$

Now, given any $\Delta>0$ and any $T>0$ let $\Delta^{*}:=\min \{\Delta, 1\}$. By setting $z_{0}=-\frac{\Delta^{*}}{\sqrt{2}}$ and

$$
y_{0}=\min \left\{\sqrt{\frac{\Delta^{*}}{2\left(e^{\frac{2 \Delta^{*} T}{\sqrt{2}}}-1\right)}} ; \frac{\Delta^{*}}{\sqrt{2}}\right\},
$$

it holds that $\left|x_{0}\right| \leq 1$ and we get from (21) that $t_{1} \geq T$. Recalling that $t^{*} \geq t_{1}$ and $y\left(t^{*}\right)=\left|x_{0}\right| \geq \Delta^{*} / \sqrt{2}$ (see (20)), we conclude that there exist $\left|x_{0}\right| \leq \Delta^{*} \leq \Delta$ and a time $t^{*} \geq T$ such that $y\left(t^{*}\right) \geq \Delta^{*} / \sqrt{2}$. Hence, for any $\varepsilon<\Delta^{*} / \sqrt{2}$, it is impossible to find $T(\varepsilon)$ that satisfies condition (6). This disproves uniformity in $x_{0}$.

Remark 6: In this counterexample, the proposed Lyapunov function is both upper and lower bounded by a function of the whole state norm (see (17)). This shows that Assertion 3 would still be untrue if (8) was replaced by the stronger requirement $a(\|\phi\|) \leq$ $V(t, \phi) \leq b(\|\phi\|)$.

Remark 7: While not required by Assertion 3, the assumptions of Assertion 4 can also be met with the following continuously differentiable Lyapunov function:

$$
V(x)=\frac{1}{2}\left(2 \sqrt{y^{2}+z^{2}}-z\right)^{2} .
$$

Then $a, b, c$ can be picked as $a(s)=(\sqrt{2}-1)^{2} s^{2} / 2$, $b(s)=9 s^{2} / 2$, and $c(s)=(\sqrt{2}-1) s^{3}$ for all $s \geq 0$.

\section{CONSEQUENCES OF LACK OF UNIFORMITY}

An obvious practical consequence of non-uniformity with respect to initial states is that transient effects, including overshoot, may happen arbitrarily late, which may be undesirable in many control applications. But this non-uniformity may also have a strong impact in terms of robustness to exogenous disturbances.

\section{A. Link between uniform asymptotic y-stability and IOS}

Using classical considerations on converse Lyapunov functions, it is possible to show that uniform asymptotic $y$-stability of an input-free system guarantees some robustness to small exogenous inputs for a small initial state. In order to make this precise, consider the system

$$
\dot{x}(t)=X(y(t), z(t), u(t)),
$$

with state $x(t)=(y(t), z(t)) \in \mathbb{R}^{n}$, output $y(t) \in \mathbb{R}^{m}$, and input $u \in \mathcal{U}^{p}$, where the vector field $X$ is assumed locally Lipschitz and $\mathcal{U}$ is the set of all measurable, locally essentially bounded signals $u: \mathbb{R}_{\geq 0} \rightarrow \mathbb{R}$. 
Let us recall the definition of input-to-output stability, originally proposed in [11].

Definition 8: Let $E \subset \mathbb{R}^{n}$ and $\mathcal{V} \subset \mathcal{U}^{p}$ be open sets. System (22) is input-to-output stable (IOS) over $E \times \mathcal{V}$ if there exist $\beta \in \mathcal{K} \mathcal{L}$ and $\gamma \in \mathcal{K}_{\infty}$ such that, for all $x_{0} \in E$ and all $u \in \mathcal{V}$,

$$
\left|y\left(t ; x_{0}, u\right)\right| \leq \beta\left(\left|x_{0}\right|, t\right)+\gamma\left(\left\|u_{[0, t]}\right\|\right), \quad \forall t \geq 0,
$$

where $u_{[0, t]}:[0, t] \rightarrow \mathbb{R}_{\geq 0}$ denotes the restriction of $u$ to the interval $[0, t]$.

The IOS property contains several ingredients. First, if $0 \in E$ and $0 \in \mathcal{V}$, then IOS over $E \times \mathcal{V}$ guarantees uniform asymptotic $y$-stability. Moreover, it guarantees that the output $y$ converges to the ball of radius $\gamma(\|u\|)$, which in turn ensures a bounded output in response to any bounded input (provided that $x_{0} \in E$ and $u \in \mathcal{V}$ ). Finally, IOS also induces the following converging input - converging output property: for all $u \in \mathcal{V}$ such that $\lim _{t \rightarrow \infty}|u(t)|=0$ and for all $x_{0} \in E$, it holds that $\lim _{t \rightarrow \infty}|y(t)|=0$. This can be shown by considering a time $T$ such that $\gamma(|u(t)|)$ remains below some arbitrary $\varepsilon>0$ for all $t \geq T$, and considering $x(T)$ as an initial state in (23), yielding: $|y(t)| \leq \beta(|x(T)|, t-T)+\varepsilon$ for all $t \geq T$; for $t$ large enough, this ensures $|y(t)| \leq 2 \varepsilon$, hence $\lim _{t \rightarrow \infty}|y(t)|=0$.

These are precious features in robustness analysis, as detailed in [10]. The following result states that, for systems evolving on a bounded set, uniform asymptotic $y$-stability of the unforced system readily ensures IOS $^{1}$.

Lemma 9: Consider a bounded set $E \subset \mathbb{R}^{n}$ and an open set $\mathcal{V} \subset \mathcal{U}^{p}$ such that $E$ is forward invariant for system (22) given any input $u \in \mathcal{V}$. If the unforced system (with $u \equiv 0$ ) is uniformly asymptotically $y$-stable on $^{2} E$, then (22) is IOS over $E \times \mathcal{V}$.

Proof: If the unforced system (with $u \equiv 0$ ) is uniformly asymptotically $y$-stable on $E$, we can conclude (reasoning as in [4, Lemma 4.5]) that there exists a function $\beta \in \mathcal{K} \mathcal{L}$ such that, for all $x_{0} \in E$

$$
\omega_{1}\left(x\left(t ; x_{0}, 0\right)\right) \leq \beta\left(\omega_{2}\left(x_{0}\right), t\right), \quad \forall t \geq 0,
$$

where $\omega_{1}(x):=|y|$ and $\omega_{2}(x):=|x|$ with $x=(y, z)$. From [13, Corollary 2], there exist $\underline{\alpha}, \bar{\alpha} \in \mathcal{K}_{\infty}$, and a smooth $V: \mathbb{R}^{n} \rightarrow \mathbb{R}_{\geq 0}$, such that, for all $x \in E$,

$$
\begin{aligned}
& \underline{\alpha}(|y|) \leq V(x) \leq \bar{\alpha}(|x|) \\
& \frac{\partial V}{\partial x}(x) X(x, 0) \leq-V(x) .
\end{aligned}
$$

\footnotetext{
${ }^{1}$ This was the basis of the results presented in our paper [8].

${ }^{2}$ Meaning that is $y$-stable and satisfies (6) for all $x_{0} \in E$.
}

Let us now compute the derivative of $V$ along the solutions of (22) with arbitrary input $u \in \mathcal{V}$ :

$$
\begin{aligned}
\frac{\partial V}{\partial x}(x) X(x, u)= & \frac{\partial V}{\partial x}(x) X(x, 0) \\
& +\frac{\partial V}{\partial x}(x)(X(x, u)-X(x, 0)) \\
\leq & -V(x) \\
& +\left|\frac{\partial V}{\partial x}(x)\right||X(x, u)-X(x, 0)| .
\end{aligned}
$$

Since $V$ is smooth and $E$ is bounded, there exists a constant $c>0$ such that $\left|\frac{\partial V}{\partial x}(x)\right| \leq c$ for all $x \in E$. Moreover, since $X$ is locally Lipschitz, there exists a continuous non-decreasing function $\ell: \mathbb{R}_{\geq 0} \rightarrow \mathbb{R}_{\geq 0}$ such that $|X(x, u)-X(x, 0)| \leq \ell(|x|+|u|)|u|$. Let $\Delta>0$ be such that $|x| \leq \Delta$ for all $x \in E$. It follows that $|X(x, u)-X(x, 0)| \leq \ell(\Delta+|u|)|u|$. Consequently:

$$
\frac{\partial V}{\partial x}(x) X(x, u) \leq-V(x)+c \ell(\Delta+|u|)|u| .
$$

Integrating this differential inequality along the solutions of (22) yields, for all $x_{0} \in E$ and all $u \in \mathcal{V}$,

$$
\begin{aligned}
V(x(t)) & \leq V\left(x_{0}\right) e^{-t}+\int_{0}^{t} e^{-(t-\tau)} c \ell(\Delta+|u(\tau)|)|u(\tau)| d \tau \\
& \leq V\left(x_{0}\right) e^{-t}+c \ell\left(\Delta+\left\|u_{[0, t]}\right\|\right)\left\|u_{[0, t]}\right\| .
\end{aligned}
$$

Using the bound (25), we get that

$$
\begin{aligned}
|y(t)| \leq & \underline{\alpha}^{-1}\left(\bar{\alpha}\left(\left|x_{0}\right|\right) e^{-t}+c \ell\left(\Delta+\left\|u_{[0, t]}\right\|\right)\left\|u_{[0, t]}\right\|\right) \\
\leq & \underline{\alpha}^{-1}\left(2 \bar{\alpha}\left(\left|x_{0}\right|\right) e^{-t}\right) \\
& +\underline{\alpha}^{-1}\left(2 c \ell\left(\Delta+\left\|u_{[0, t]}\right\|\right)\left\|u_{[0, t]}\right\|\right),
\end{aligned}
$$

where we used the fact that $\underline{\alpha}^{-1}(a+b) \leq \underline{\alpha}^{-1}(2 a)+$ $\underline{\alpha}(2 b)$ for all $a, b \in \mathbb{R}_{\geq 0}$ since $\underline{\alpha}^{-1} \in \mathcal{K}_{\infty}$. Defining $\beta(s, t):=\underline{\alpha}^{-1}\left(2 \bar{\alpha}(s) e^{-t}\right)$ and $\gamma(s):=$ $\underline{\alpha}^{-1}(2 c \ell(\Delta+s) s)$ for all $s, t \in \mathbb{R}_{\geq 0}$, we finally obtain:

$$
|y(t)| \leq \beta\left(\left\|x_{0}\right\|, t\right)+\gamma\left(\left\|u_{[0, t]}\right\|\right),
$$

for all $x_{0} \in E$ and all $u \in \mathcal{V}$. IOS follows by observing that $\beta \in \mathcal{K} \mathcal{L}$ and $\gamma \in \mathcal{K}_{\infty}$.

\section{B. Importance of uniformity in IOS analysis}

Lemma 9 shows that, for systems evolving on a bounded set, $y$-UAS in the absence of inputs ensures IOS. This constitutes a handy way to guarantee robustness to exogenous inputs and was actually at the basis of the reasoning made in our recent paper [8] (although in a time delay context). The following example ${ }^{3}$ shows that

\footnotetext{
${ }^{3}$ The proposed example has the inelegant feature that the state converges to the boundary of $E$. However, it has the advantage of being concise and easy to grasp.
} 
this relationship is not valid anymore if the uniformity requirement is not fulfilled.

Proposition 10: Consider the bi-dimensional system

$$
\begin{aligned}
& \dot{y}=-z y+u \varphi(y) \\
& \dot{z}=-z^{2}
\end{aligned}
$$

where $\varphi: \mathbb{R} \rightarrow \mathbb{R}$ is any smooth function satisfying

$$
\varphi(y)= \begin{cases}1 & \text { if }|y| \leq 1 \\ 0 & \text { if }|y| \geq 2\end{cases}
$$

Let $E:=(-2 ; 2) \times(0 ; 1)$. Then the following holds:

i) System (26) is 0-y-AS. More precisely, for all $x_{0}=\left(y_{0}, z_{0}\right) \in E$, its solution for $u \equiv 0$ satisfies $|x(t)| \leq\left|x_{0}\right|$ for all $t \geq 0$ and $\lim _{t \rightarrow+\infty}|y(t)|=0$.

ii) Given any $u \in \mathcal{U}$, the bounded set $E$ is forward invariant for (26).

iii) For any $\left(y_{0}, z_{0}\right) \in(-1 ; 1) \times(0 ; 1)$, the input $u \in$ $\mathcal{U}$ defined as $u(t):=\frac{z_{0} y_{0}}{1+z_{0} t}$ converges to zero but generates a non-vanishing solution (namely, $y(t)=$ $y_{0}$ for all $t \geq 0$ ).

Proof: Let us prove the three items separately.

Item $i)$ : Given any $x_{0}=\left(y_{0}, z_{0}\right) \in E$, the solution of $\overline{(26) \text { for }} u \equiv 0$ reads $y(t)=\frac{y_{0}}{1+z_{0} t}$ and $z(t)=\frac{z_{0}}{1+z_{0} t}$. Item $i$ ) then readily follows.

Item $i i)$ : Consider any $\left(y_{0}, z_{0}\right) \in E$ and any $u \in \mathcal{U}$. Since $z(t)=\frac{z_{0}}{1+z_{0} t}$ is the solution of (26b), $z(t)>0$, and $z(t) \leq z_{0}$ for any $t \geq 0$. Thus, for any $z_{0} \in(0,1)$, we conclude that $z(t) \in(0 ; 1)$ for all $t \geq 0$.

Moreover, assume by contradiction that there exists $t>0$ such that $y(t)=2$. Pick $t^{\star}>0$ as the smallest $t$ with such a property. Since $\varphi\left(y\left(t^{\star}\right)\right)=0$ and $z\left(t^{\star}\right)>0$, it follows that $\dot{y}\left(t^{\star}\right)=-2 z\left(t^{\star}\right)<0$, contradicting the minimality of $t^{\star}$ among the times at which $y(t)=2$. Proceeding similarly for $y(t)=-2$, we conclude that $y(t) \in(-2 ; 2)$ for all $t \geq 0$.

Item $i$ ii $)$ : We claim that $y(t)=y_{0}$ and $z(t)=\frac{z_{0}}{1+z_{0} t}$ is the solution for that particular input. We have already noticed that $z(t)$ solves (26b). Equation (26a) is also satisfied, since

$-z(t) y(t)+u(t) \varphi(y(t))=-\frac{z_{0}}{1+z_{0} t} y_{0}+\frac{z_{0} y_{0}}{1+z_{0} t}=0$

where we used that $\varphi\left(y_{0}\right)=1$ (recall that $\left|y_{0}\right|<1$ ).

System (26) is thus an example of a system which is $y$-AS in the absence of an input (although not uniformly), whose solutions evolve on a bounded open set, but for which the converging input - converging output property does not hold. Since the latter property is a natural consequence of IOS (as recalled above), this example illustrates why the uniformity requirement with respect to initial states is instrumental in the analysis of robustness with respect to exogenous disturbances.

Since [14, Theorem 6.2.1] does not guarantee this uniformity (Section III), its relevance in the context of robustness analysis is compromised.

\section{Conclusion}

We have disproved a Lyapunov sufficient condition for partial stability. More precisely, we have shown that uniformity of the convergence with respect to initial states may not hold. We have also underlined through an example how crucial this uniformity is in the study of robustness with respect to exogenous inputs. The results in this paper invalidate our recent publication [8], which studied adaptive control for nonlinear time-delay systems: a correction will soon be provided.

\section{ACKNOWLEDGMENT}

We would like to thank E. Panteley, I. Karafyllis, and F. Mazenc for fruitful discussions about the issues considered in this paper.

\section{REFERENCES}

[1] P. Ioannou and P. Kokotovic. Instability analysis and improvement of robustness of adaptive control. Automatica, 20(5):583594, 1984.

[2] H. G. Kankanamalage, Y. Lin, and Y. Wang. On LyapunovKrasovskii characterizations of input-to-output stability. IFACPapersOnLine, 50(1):14362-14367, 2017.

[3] I. Karafyllis, P. Pepe, and Z.-P. Jiang. Input-to-output stability for systems described by retarded functional differential equations. Eur. J. Control, 14(6):539-555, 2008.

[4] H. Khalil. Nonlinear systems. Prentice Hall, 3rd ed., New York, 2002.

[5] N. Krasovskii. Stability of motion. Stanford Univ. Press, 1963.

[6] V. Lakshmikantham and X. Liu. Stability analysis in terms of two measures. World Scientific, 1993.

[7] J. L. Massera. Contributions to stability theory. Annals of Mathematics, pages 182-206, 1956.

[8] J. Orlowski, A. Chaillet, M. Sigalotti, and A. Destexhe. Adaptive scheme for pathological oscillations disruption in a delayed neuronal population model. In 2018 IEEE Conference on Decision and Control (CDC). IEEE, Dec. 2018.

[9] A. Oziraner and V. Rumiantsev. The method of Liapunov functions in the stability problem for motion with respect to a part of the variables. Prikl. Mat. Meh., 36:364-384, 1972.

[10] E. Sontag. Input to state stability: Basic concepts and results, pages 163-220. Lecture Notes in Mathematics. Springer-Verlag, Berlin, 2008.

[11] E. Sontag and Y. Wang. Notions of input-to-output stability. Systems \& Contol Letters, 38:235-248, 1999.

[12] E. Sontag and Y. Wang. Lyapunov characterizations of input to output stability. SIAM J Control Optim., 39(1):226-249, 2000.

[13] A. R. Teel and L. Praly. A smooth Lyapunov function from a class- $\mathscr{K} \mathscr{L}$ estimate involving two positive semidefinite functions. ESAIM Control Optim. Calc. Var., 5:313-367, 2000.

[14] V. Vorotnikov. Partial stability and control. Birkhauser, 1998.

[15] V. Vorotnikov. Partial stability and control: The state-of-the-art and development prospects. Automation and Remote Control, 66(4):511-561, 2005. 